\title{
NOTE ON THE COHOMOLOGY GROUPS OF ASSOCIATIVE ALGEBRAS
}

\author{
HIROSI NAGAO
}

The cohomology theory of associative algebras has been cieveloped by $G$. Ifochschild [1], [2], [3], and the 1-, 2-, and 3-dimensional cohomology groups have been interpreted with reference to classical notions of structure in his papers. Recently M. Ikeda has obtained, by a detailed analysis of Hochschild's modules, an interesting structural characterization of the class of algebras whose ¿-dimensional cohomology groups are all zero [5].

In sections 1 and 2 , we consider an algebra whose residue class algebra rodulo its radical is separable, and offer a criterion for such algebra to have trivial $n(\geqslant 2)$-dimensional cohomology group in terms of certain module, which is similar to Hochschild's module but is rather simpler.

In section 3 , we consider the cases of dimensions 2 and 3 . We offer another proof of Ikeda's theorem, and, under the assumpion that $A / N$ ( $N$ is the radical of $A$ ) is separable, a structural characterization of the class of algebras whose 3-dimensional cohomology groups are all zero.

The writer wishes to express his best thanks to Prof. T. Nakayama for his kind encouragement and advice, and to Mr. M. Ikeda for his discussions and suggestions during the preparation of this note.

1. Let $A$ be an associative algebra over a field $F$ which possesses a unit element 1 , and $N$ be its radical. We assume, throughout this and the next section, that $A / N$ is separable. Since 2-dimensional cohomology groups of $A / N$ are all zero, $A$ contains a subalgebra $\bar{A}$ such that $A$ is decomposed into the direc: (module) sum of $\bar{A}$ and $N: A=\bar{A}+N$. Evidently $\bar{A}$ is an algebra isomorphic to $A / N$, and hence separable. We denote elements of $\bar{A}$ by $\bar{a}, \bar{b}$, $\ldots$ and those of $N$ by $m_{1}, m_{2}, \ldots$.

With an $A$ - $A$-module $\mathfrak{n}$ and a natural number $n$ we denote, after Hochschild, the modules of all $n$-cochains, $n$-cocycles, $n$-coboundaries of $A$ in $\mathfrak{n}$ by $C^{n}(A$, $\mathfrak{n}), Z^{n}(A, \mathfrak{n}), B^{n}(A, \mathfrak{n})$ respectively, and $n$-dimensional cohomology group of $A$ in $\mathrm{n}$ by $H^{n}(A, \mathfrak{n})$.

Let $P_{n}=A \times \ldots \times A$ be the $n$-fold direct product of the underlying vector space of $A$. We define the operations on $P_{n}$ by setting

Received July 1, 1953. 


$$
\left\{\begin{array}{l}
a_{0} *\left(a_{1} \times \ldots \times a_{i 2}\right)=\sum_{i=0}^{n-1}(-1)^{i} a_{0} \times \ldots \times a_{i} a_{i+1} \times \ldots \times a_{n}, \\
\left(a_{1} \times a_{2} \times \ldots \times a_{n}\right) * a_{n+1}=a_{1} \times a_{2} \times \ldots \times a_{n} a_{n+1} .
\end{array}\right.
$$

This makes $P_{n}$ an $A$-A-module. ${ }^{1)}$ We call this the $n$-aimensional Hochschild module of $A$.

Lemma 1.1. Let $\mathfrak{n}$ be an $A$-A-module. If $f$ is an element of $C^{n}(A, \mathfrak{n})$ and of $\left(\bar{a}_{1}, a_{2}, \ldots, a_{n+1}\right)=0$ for any element $\bar{a}_{1}$ of $\bar{A}$, then there exists an element $g$ of $C^{n-1}(A, \mathfrak{n})$ such that $(f-\delta g)\left(\bar{a}_{1}, a_{2}, \ldots, a_{n}\right)=0$ for any element $\bar{a}_{1}$ of $\bar{A}$.

Proof. Let $R\left(P_{n}, \mathfrak{n}\right)$ be the module of all right operator homomorphisms from $P_{n}$ into $n$. We define the operations of the elements of $A$ for $F \in R\left(P_{n}\right.$, n) by setting

$$
\begin{aligned}
& (a \circ F)\left(a_{1} \times a_{2} \times \ldots \times a_{n}\right)=a F\left(a_{1} \times a_{2} \times \ldots \times a_{n}\right), \\
& (F \circ a)\left(a_{1} \times a_{2} \times \ldots \times a_{n}\right)=F\left(a *\left(a_{1} \times a_{2} \times \ldots \times a_{n}\right)\right) .
\end{aligned}
$$

Under these operations, $R\left(P_{n}, \mathfrak{n}\right)$ is an $A$ - $A$-module.

For an $f \in C^{n}(A, n)$ having the property in the lemma we define an element $F(f)$ of $C^{1}\left(\bar{A}, R\left(P_{n}, \mathfrak{n}\right)\right)$ by the relation $F(f)\left(\bar{a}_{1}\right)\left(a_{2} \times \ldots \times a_{n+1}\right)=f\left(\bar{a}_{1}, a_{2}\right.$, $\left.\ldots, a_{n}\right) a_{n+1}$. Then we can verify, from the property of $f$, that $\delta F(f)=0$. Since $\bar{A}$ is separable, there exists an element $G$ of $R\left(P_{n}, n\right)$ such that $F^{\prime}(f)(\bar{a})$ $=\delta G(\bar{a})=\bar{a} \circ G-G \circ \bar{a}$. We define $g \in C^{n-1}(A, \mathfrak{n})$ by setting

$$
g\left(a_{1}, a_{2}, \ldots, a_{n-1}\right)=G\left(a_{1} \times a_{2} \times \ldots \times a_{n-1} \times 1\right),
$$

then we see, from the property of $G$, that $g$ satisfies the requierment of the lemma.

Now let $Q_{n-1}=N \times A \times \ldots \times A$ be the direct product of the vector spaces of $N$ and $(n-2)$-fold direct product of $A$. We define the operations of the element of $A, \bar{A}$ on $Q_{n-1}$, on the right and left sides, respectively, by setting

$$
\left\{\begin{array}{l}
\left(m_{1} \times a_{2} \times \ldots \times a_{n-1}\right) * a_{n}=\sum_{i=1}^{n-1}(-1)^{n-i-1} m_{1} \times \ldots \times a_{i} a_{i+1} \times \ldots \times a_{n}, \\
\bar{a}_{0} *\left(m_{1} \times a_{2} \times \ldots \times a_{n-1}\right)=\bar{a}_{0} m_{1} \times a_{2} \times \ldots \times a_{n-1} .
\end{array}\right.
$$

This makes $Q_{n-1}$ an $\bar{A}$ - $A$-module.

We denote by $\bar{L}\left(Q_{n-1}, \mathfrak{n}\right)$ the module of all $\bar{A}$-(left) operator homomorphisms from $Q_{n-1}$ into $\mathfrak{n}$, and define the operations of the elements of $A$ for $F \in \bar{L}\left(Q_{n-1}, \mathfrak{n}\right)$ by setting

$$
\left\{\begin{array}{l}
(a \circ F)\left(m_{1} \times a_{2} \times \ldots \times a_{n-1}\right)=F\left(\left(m_{1} \times a_{2} \times \ldots \times a_{n-1}\right) * a\right) \\
(F \circ a)\left(m_{1} \times a_{2} \times \ldots \times a_{n-1}\right)=F\left(m_{1} \times a_{2} \times \ldots \times a_{n-1}\right) a .
\end{array}\right.
$$

1) A module $\mathfrak{m}$ is called an $A$ - $A$-module if $\mathfrak{m}$ is $A$-left and right module and satisfies $a(m b)$ $=(a m) b(a, b \in A, m \in \mathfrak{M l})$. 
Under these operations $\bar{L}\left(Q_{n-1}, \mathfrak{n}\right)$ is an $A$-A-module.

ThEOREM 1.1. Let $\mathfrak{n}$ be a module such that $N \mathfrak{n}=\mathfrak{n} N=0$. Then (under the assumption that $A / N$ is separable)

$$
H^{n}(A, \mathfrak{n}) \simeq H^{1}\left(A, \tilde{L}\left(Q_{n-1}, \mathfrak{n}\right)\right) \quad(n \geq 2) .
$$

Proof. Denote by $\bar{C}^{n}(A, \mathfrak{n})$ the module of all $n$-cochains $f$ such that $f\left(a_{1}\right.$, $\left.a_{2}, \ldots, a_{n}\right)=0$ for any element $\bar{a}_{1}$ of $\bar{A}$, and set $\bar{Z}^{n}(A, \mathfrak{n})=Z^{n}(A, \mathfrak{n}) \frown \bar{C}^{n}(A$, $\mathfrak{n}), \bar{B}^{n}(A, \mathfrak{n})=B^{n}(A, \mathfrak{n}) \frown \bar{C}^{n}(A, \mathfrak{n})$. From Lemma 1.1 every cohomology class contains an element of $\bar{Z}^{\prime \prime}(A, \mathfrak{n})$, and hence $H^{n}(A, \mathfrak{n})$ is isomorphic to $\bar{Z}^{n}(A$, n) $/ \bar{B}^{n}(A, \mathfrak{n})$. With an element $f$ of $\bar{Z}^{\prime \prime}(A, \mathfrak{n})$ and an element $a_{n}$ of $A$, we define a linear mapping $F(f)\left(a_{n}\right)$ from $Q_{n-1}$ into $n$ by the relation $F(f)\left(a_{n}\right)$ $\left(m_{1} \times a_{2} \times \ldots \times a_{\eta_{-1}}\right)=f\left(m_{1}, a_{2}, \ldots, a_{n}\right)$. Since $\delta f\left(\bar{a}, m_{1}, a_{2}, \ldots, a_{n}\right)=\bar{a} f\left(m_{1}\right.$, $\left.a_{2}, \ldots, a_{n}\right)-f\left(a m_{1}, a_{2}, \ldots, a_{n}\right)=0, F(f)\left(a_{n}\right)$ is an element of $L\left(Q_{n-1}, \mathfrak{n}\right)$ and $F(f)$ is an element of $C^{1}\left(A, \bar{L}\left(Q_{n-1}, \mathfrak{n}\right)\right)$. Taking account of the assumed property of $n$ we see by direct computations that $\left(\delta F(f)\left(a_{n}, a_{n+1}\right)\right)\left(m_{1} \times \ldots\right.$ $\left.\times a_{n-1}\right)=\delta f\left(m_{1}, a_{2}, \ldots, a_{n+1}\right)=0$, and hence $F(f) \in Z^{1}\left(A, \bar{L}\left(Q_{n-1}, n\right)\right)$.

Now let $f$ be an element of $\bar{B}^{n}(A, \mathfrak{n})$. Then there exists an element $g^{\prime}$ of $C^{n-1}(A, \mathfrak{n})$ such that $f=\delta g^{\prime}$. Since $\delta g^{\prime}\left(\bar{a}_{1}, a_{2}, \ldots, a_{n}\right)=0$ for $\bar{a}_{1} \in \bar{A}$, from Lemma 1.1 there exists an element $h$ of $C^{n-2}(A, \mathfrak{n})$ such that $\left(g^{\prime}-\delta h\right)\left(\bar{a}_{1}\right.$, $\left.a_{2}, \ldots, a_{n-1}\right)=0$ for $\bar{a}_{1} \in \bar{A}$. Set $g=g^{\prime}-\delta h$, then $f=\delta g$ and $g \in \bar{C}^{n-1}(A, n)$. Since $f\left(\bar{a}_{0}, m_{1}, a_{2}, \ldots, a_{n-1}\right)=o g\left(\bar{a}_{0}, m_{1}, a_{2}, \ldots, a_{n-1}\right)=\bar{a}_{9} g\left(m_{1}, a_{2}, \ldots\right.$ $\left.a_{n-1}\right)-g\left(a_{0} m, a_{2}, \ldots, a_{n-1}\right)=0$, if we set $G\left(m_{1} \times a_{2} \times \ldots \times a_{n-1}\right)=g\left(m_{1}, a_{2}\right.$, $\left.\ldots, a_{n-1}\right)$ then $G \in L\left(Q_{n-1}, \mathfrak{n}\right)$. By direct computations we can verify that $F(f)(a)= \pm \delta G$, and hence the mapping $f \rightarrow F(f)$ induces a homomorphism from $H^{n}(A, \mathfrak{n})$ into $H^{1}\left(A, L\left(Q_{n-1}, \mathfrak{n}\right)\right)$.

Conversely, if $F$ is an element of $Z^{1}\left(A, L\left(Q_{n-1}, \mathfrak{n}\right)\right)$ we define an element $f$ of $\bar{C}^{n}(A, n)$ by setting

$$
\begin{aligned}
& f\left(\bar{a}_{1}, a_{2}, \ldots, a_{n}\right)=0 \text { for } \bar{a}_{1} \in \bar{A}, \\
& f\left(m_{1}, a_{2}, \ldots, a_{n}\right)=F\left(a_{n}\right)\left(m_{1} \times \ldots \times a_{n-1}\right) \text { for } m_{1} \in N .
\end{aligned}
$$

Then it is easily seen that $f$ is an element of $\bar{Z}^{n}(A, \mathfrak{n})$ and $F=F(f)$. This shows that $H^{\prime \prime}(A, \mathfrak{r})$ is mapped onto $H^{1}\left(A, L\left(Q_{n-1}, \mathfrak{n}\right)\right)$ by the above mapping. Further if $F(f)$ is a coboundary, that is, $F(f)=\delta G$, then we see that $f=\delta g$, where $g$ is an element of $C^{n-1}(A, \mathfrak{n})$ defined by the relations $g\left(m_{1}, a_{2}, \ldots, a_{n-1}\right)$ $=G\left(m_{1} \times a_{2} \times \ldots \times a_{n-1}\right)$, for $m_{1} \in N$, and $g\left(\bar{a}_{1}, a_{2}, \ldots, a_{n-1}\right)=0$, for $\bar{a}_{1} \in A$. This shows that the above homomorphism is an isomorphism.

2. In this section, we recall some definitions and properties about the module extensions and offer a criterion for $A$ to have trivial $n$-dimensional cohomology groups in terms of $Q_{n-1}$.

Let $m$ and $\mathfrak{n}$ be two modules with the same operator domain $\Omega$. We call 
a third $\Omega$-module $\mathfrak{M}$ an $(\Omega$-)extension of $\mathfrak{n}$ by $\mathfrak{m}$ if $\mathfrak{M}$ contains $\mathfrak{n}$ and $\mathfrak{M} / \mathfrak{n} \cong \mathfrak{m}$. If an extension $\mathfrak{M}$ of $n$ by $m$ contains an $\left(\Omega\right.$-) submodule $m^{\prime}$ such that $m$ is the direct sum $\mathfrak{R}=\mathfrak{n}+\mathfrak{m}^{\prime}$, then we say that $\mathfrak{m}$ splits. If for any $\Omega$-module $\mathfrak{n}$ every extension of $\mathfrak{n}$ by $m$ splits, we call $m$ an $\left(M_{0}\right)$-module.

Now let $m$ and $\mathfrak{n}$ be two $\bar{A}-A$-modules and $\mathfrak{M}$ be an $(\bar{A}-A$-)extensions of $\mathrm{n}$ by $\mathrm{m}$. For $u \in m$, take a system of linear representatives $\left\{B_{u}\right\}$. Then

$$
\begin{cases}\bar{a} B_{u}=B_{\bar{a} u}+\hat{\beta}(\bar{a}, u) & (\bar{a} \in \bar{A}, \beta(\bar{a}, u) \in \dot{\mathfrak{n}}), \\ B_{u} a=B_{u a}+\gamma(u, a) & (a \in A, \gamma(u, a) \in \mathfrak{n}) .\end{cases}
$$

$\beta(\bar{a}, u)$ and $\gamma(u, a)$ are linear in $\bar{a}, a, u$. From the associative relations $\bar{a}\left(\bar{b} B_{u}\right)=(\bar{a} \bar{b}) B_{u}, \quad\left(\bar{a} B_{u}\right) b=\bar{a}\left(B_{u} b\right),\left(B_{u} a\right) b=B_{u}(a b)$, we have

$$
\left\{\begin{array}{l}
\bar{a} \beta(\bar{b}, u)+\beta(\bar{a}, \bar{b} u)-\beta(\bar{a} \bar{b}, u)=0, \\
\beta(\bar{a}, u b)-\beta(\bar{a}, u) b=\gamma(\bar{a} u, b)-\bar{a} \gamma(u, b), \\
\gamma(u, a) b+\gamma(u a, b)-\gamma(u, a b)=0 .
\end{array}\right.
$$

The structure of $\mathfrak{M}$ is completely determined by $\{\beta, \gamma\}$, and conversely if $\{\beta, \gamma\}$ sutisfies the relations (5) we have an extension of $\mathfrak{n}$ by $\mathfrak{m}$, by (4). We call $\{\beta, \gamma\}$ satisfying (5) a factor system. Two factor systems $\left\{\beta_{1}, \gamma_{1}\right\}$ and $\left\{\beta_{2}, \gamma_{2}\right\}$ are called associated if there exists a linear mapping $\lambda$ from $\mathfrak{m}$ into $\mathfrak{n}$ satisfying the relations

$$
\left\{\begin{array}{l}
\beta_{2}(\bar{a}, u)=\beta_{1}(\bar{a}, u)+\{\bar{a} \lambda(u)-\lambda(\bar{a} u)\} \\
\gamma_{2}(u, a)=\gamma_{1}(u, a)+\{\lambda(u) a-\lambda(u a)\}
\end{array}\right.
$$

As is well known, $\left\{\beta_{1}, \gamma_{1}\right\}$ and $\left\{\beta_{2}, \gamma_{2}\right\}$ are associated if and only if they define equivalent extensions. ${ }^{2\rangle}$

We denote by $\bar{L}(m, \mathfrak{n})$ the module of all $\bar{A}$-(left) operator homomorphisms from $m$ into $n$, and, defining the operations as (3), we make this an $A$ - $A$-module. Since every $\left(\bar{A}-A^{-}\right)$extension of $\mathrm{n}$ by $\mathrm{m}$ is $(\bar{A}-)$ left inessential, ${ }^{3)}$ by an argument similar to those in [3] or [6], we can verify the following lemma.

Lemma 2.1. Let $\amalg$ and $\mathfrak{n}$ be two $\bar{A}-A$-modules. Then all extensions of $\mathfrak{n}$ by $\mathrm{m}$ split if and only if $H^{1}(A, \bar{L}(\mathrm{~m}, \mathfrak{n}))=0$.

Let next

$$
\bar{A}=\sum_{\kappa=1}^{k} \bar{A} e_{\kappa}=\sum_{\kappa=1}^{k} e_{\kappa} \bar{A}
$$

be direct decompositions of $\bar{A}$ into indecomposable left and right ideals, and

$\Rightarrow$ Two extensions $\mathfrak{M}_{1}$, $\mathfrak{M}_{2}$ of $\mathrm{n}$ by $\mathfrak{m}_{\mathrm{t}}$ are called equivalent if there exists an isomorphism between $\mathfrak{M}_{1}$ and $\mathfrak{M}_{2}$ which leaves invariant each element of $\mathfrak{n}$ as well as the isomorphism from $m_{i} / \mathrm{n}$ to $\mathrm{m}$.

2) An $\bar{A} \cdot A$-extension $9 i$ of $u$ by $\mathfrak{i n}$ is called $(\bar{A}-)$ left inessential if $M_{i}$ splits as an $\bar{A}$-(left) extension. 
$\left\{e_{\kappa}\right\}$ be mutually othogonal primitive idempotents. Then

$$
A=\sum_{\kappa=1}^{k} A e_{\kappa}=\sum_{\kappa=1}^{k} e_{\kappa} A
$$

are direct decompositions of $A$ into indecomposable left and right ideals.

The structure theorem of $\left(M_{0}\right)$-modules states (see [7]):

Lemma 2.2. An A-right module $\mathrm{m}$ is an $\left(M_{0}\right)$-module if and only if $1 \mathrm{in}$ is a direct sum of submodules isomorphic to $e_{\mathrm{K}} A$.

Now we have

Lemma 2.3. Let $\mathrm{m}$ be an $\bar{A}$-A-moálule, and suppose that $1 u=u$ for $u \in \|$. $\mathrm{ni}$ is an $\left(M_{0}\right)$-module as an $\bar{A}-A$-inoalule if and only if it is so as an $A$-(right) module.

Proof. i) Let $\mathrm{m}$ be an $\left(M_{0}\right)$-module as an $\bar{A}$ - $A$-module. Then $1 m 1=m 1$ is a direct sum of submodules isomorphic to $\bar{A} e_{\mathrm{\kappa}} \times e_{\lambda} A$, and hence as $A$-right module directly decomposed into a direct sum of submodules isomorphic to $e_{\lambda} A$. This show that $m$ is an $\left(M_{0}\right)$-module as $A$-rigint module.

ii) Let $m$ be an $\left(M_{0}\right)$-module as $A$-right module. It is sufficient to prove that for any $\bar{A}-A$-module $n$ such that $n N=0$, every extension of $n$ by $i$ splits. Let $\mathfrak{n}$ be such a module, and $\{\beta, \gamma\}$ a factor system. Since $A$ is separable, ive can assume that $\beta(\bar{a}, u)=\gamma(u, \bar{a})=0$. Then $\{\beta, \gamma\}$ satisfies the relations

$$
\left\{\begin{array}{c}
\text { i) } \hat{\beta}(\bar{a}, u)=r(u, \bar{a})=0, \\
\text { ii) } \gamma(\bar{a} u, m)-\bar{a} \gamma(u, m)=0, \\
\text { iii) } r(u, m) \bar{b}-r(u, m \bar{b})=0, \\
\text { iv) } r(u \bar{a}, m)-r(u, \bar{a} m)=0 .
\end{array}\right.
$$

And the extension determined by $\{\beta, \gamma\}$ splits if and only if there exists a linear mapping $\lambda$ from $m$ into $n$ satisfying the relations

$$
\left\{\begin{array}{l}
\beta(\bar{a}, u)=\bar{a} \lambda(u)-\lambda(\bar{a} u)=0 \\
r(u, \bar{a})=\lambda(u) \bar{a}-\lambda(u \bar{a})=0 \\
\gamma(u, m)=-\lambda(u \dot{m}) .
\end{array}\right.
$$

Since $\mathrm{m}$ is an $\left(M_{0}\right)$-module as an $A$-right module, there exists a linear mapping $\lambda^{\prime}$ satisfying the relations

$$
\left\{\begin{array}{l}
\gamma(u, \bar{a})=\lambda^{\prime}(u) \bar{a}-\lambda^{\prime}(u \bar{a})=0, \\
\gamma(u, m)=-\lambda^{\prime}(u m) .
\end{array}\right.
$$

Now, since $m$ is completely reducible as $\bar{A}-\bar{A}$-module, $m$ is decomposed into a direct sum of $m N$ and an another $\bar{A} \cdot \bar{A}$-submodule $m_{0} ; m=m N+m_{0}$. From (7) ii) and iii), $\lambda^{\prime}$ induces an $\bar{A} \cdot \bar{A}$-operater homomorphism from $m N$ into :. Hence if we deine a mapping $\lambda$ from $m$ into $n$ by setting 


$$
\begin{aligned}
& \lambda(u m)=\lambda^{\prime}(u m), \\
& \lambda\left(u u_{0}\right)=0 \text { for } u_{0} \in \mathbb{m}_{0},
\end{aligned}
$$

then $\lambda$ satisnies the relations (8), and the extension determined by $\{\beta, \gamma\}$ splits.

Lemma 2.4. $H^{n}(A, \mathfrak{n})=0$ for every $A$-A-module $\mathfrak{n}$ if (and only if) it holds for every $A$-A-module $\mathfrak{n}$ such that $N \mathfrak{n}=\mathrm{n} N=0$.

Proof. Suppose that $H^{n}(A, \mathfrak{n})=0$ for all $\mathfrak{n}$ such that $N \mathfrak{n}=n N=0$. Let $m$ be an $A$-A-module and $\mathrm{m}=\mathrm{m}_{0} \supset \mathrm{m}_{1} \supset \mathrm{m}_{1} \supset \ldots \supset \mathrm{m}_{t}=0$ be a composition series of $m$. In case $t=1, N m=\mathfrak{m} N=0$ and hence $H^{n}(A, \mathfrak{m})=0$. Now suppose that $H^{n}(A, \mathfrak{n})=0$ for all $\mathfrak{n}$ with a length of composition series less than $t$, and consider an $f \in Z^{n}(A, \mathrm{~m})$. Set $\bar{f}\left(a_{1}, \ldots, a_{n}\right) \equiv f\left(a_{1}, \ldots, a_{n}\right)$ mod $\mathrm{m}_{t-1}$, then $\bar{f} \in Z^{n}\left(A, \mathrm{~m} / \mathrm{m}_{t-1}\right)$. Since the length of conposition series is equal to $t-1$, $\vec{f} \in B^{n}\left(A, \mathrm{~m} / \mathrm{m}_{t-1}\right)$. Hence, there exists an element $g_{1}$ of $C^{n-1}(A, m)$ such that $\bar{f}\left(a_{1}, \ldots, a_{n}\right) \equiv \delta g_{1}\left(a_{1}, \ldots, a_{n}\right) \bmod m_{t-1}$. Since $f-\delta g_{1} \in Z^{n}\left(A, \mathfrak{m}_{t-1}\right)$ and $N \mathrm{~m}_{t-1}=\mathfrak{m}_{t-1} N=0$, there exists a $g_{2} \in C^{n-1}\left(A, \mathrm{~m}_{t-1}\right)$ such that $f-\delta g_{1}=\delta g_{2}$. This shows that $f \in B^{n}(A, \mathrm{~m})$, and hence $H^{n}(A, \mathrm{~m})=0$.

By an argument similar to those in the above proof, we have

LemMa 2.5. An A-right module $m$ is an $\left(M_{1}\right)$-risodale if (and only if), for any A-right module $\mathfrak{n}$ such that $\mathrm{n} N=0$, all extensions of $\mathfrak{n}$ by $m$ split.

Now, from Theorem 1.1, Lemmas 2.1,2.3, 2.4, and 2.5, we have immediately the following theorem.

THEOREx 2.1. (Under the assumption that $A / N$ is separable ${ }^{4)}$ all n-dimensional cohomology grouts of $A$ are zero if and only if $Q_{n-1}$ is an $\left(M_{0}\right)$ module as an A-right moaiule.

3. In this section, we shall consider the cases of dimension 2 and 3 .

It was shown in [1] that the class of algebras whose 2-dimensional cohomology groups are all zero coinsides with the class of absolutely segregated algebras.

Since $Q_{1}$ is isomorphic to $N$ as an $A$-right module, we have immediately the following theorem, which is a special case of Ikeda's theorem.

Theorem 3.1. Let $A$ be an algebra such that $A_{i} N$ is seperable. Then $A$ is absolutely segregated if and only if $N$ is an $\left(M_{0}\right)$-module as an A-right module.

In order to prove the seperability of $A / N$ for an absolutely segregated algebra $A$, we mention the following lemina.

LeNimA 3.1. If an algebra A over an algebraically closed field $F$ is ajsolutely segregated the in the rank of $e_{\kappa} A e_{\kappa}$ over $F$, denoted by $\left[e_{\kappa} A e_{\kappa}\right]$, is equal to 1 .

Proof. Since $F$ is algebraically closed, $A / N$ is seperable. From theorem 1) Cr. a note at the end. 
3. $1, N$ is an $\left(M_{0}\right)$-module as an $A$-right module.

Let $t_{\kappa \lambda}$ be the number of factors isomorphic to $e_{\lambda} A$ in a direct decomposition of $e_{\kappa} N$ into directly indecomposable submodules: $e_{\kappa} N \cong \sum_{\lambda} t_{\kappa} e_{\lambda} A$. We assume that the inciices are so arranged as $\left[e_{1} A\right] \leqq\left[e_{z} A\right] \leqq \ldots \leqq\left[e_{k} A\right]$. Then $\kappa<\lambda$ implies $t_{\kappa \lambda}=0$. Set $c_{\kappa \lambda}=\left[e_{\kappa} A e_{\lambda}\right], C=\left(c_{\kappa \lambda}\right)$, and $T=\left(t_{\kappa \lambda}\right)$. From $e_{\kappa} N e_{\lambda}$ $\cong \sum_{\mu} t_{\kappa \mu} e_{\mu} A e_{\lambda}$, we have

$$
C(E-T)=E \quad(E: \text { unit matrix }) .
$$

Since the matrix $E-T$ is

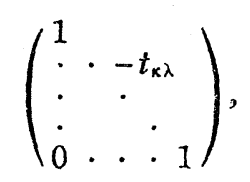

its inverse matrix $C$ is of from

$$
\left(\begin{array}{ccc}
1 & & \\
\cdot & c_{\kappa \lambda} & \\
\cdot & \cdot & \\
\cdot & & \cdot \\
0 & \cdot & \cdot
\end{array}\right)
$$

This shows that $c_{\kappa \kappa}=\left[e_{\kappa} A e_{\kappa}\right]=1$.

As was shown in the proof of "only if" part of Theorem in $\$ 5$ of [5], it is concluded rather easily from lemma 3.1 that $A ! N$ is separable if $A$ is an absolutely segregated algebra. Combining this fact with Theorem 3.1 we have immediately

Theorem 3.2. (Ikeda's Theorem). An algebra u'ith unit clement is absolutely segregated if and oinly if

i) $A / N$ is separable,

ii) $N$ is an $\left(M_{0}\right)$-mociule as A-right module.

Next, supposing that $A / N$ is separable, we consider the case of dimension 3 . Let $N \triangleq A$ be a direct product of underlying vector spaces of $N$ and $A$, and define the operation for $m \in b \in A$, as usual. by setting

$$
(m \otimes b) a=m \otimes b a \text {. }
$$

Then $N \otimes A$ is an $A$-right module. The mapping $m \& b \rightarrow m b$ induces an $A$ (right) operator homomorphism from $N \otimes A$ on $N$. We denote its kernel by $N_{0}$. Then we have

Lemma 3.1. $Q_{2} * 1 \cong N_{0}$ (as A-right modules).

Proof. Since $(m \times a) * 1=m \times a-m a \times 1, m \times a$ is contained in $Q_{2} * 1$ if and oniy if $m a=0$. If $m \times b \in Q_{2} * 1$, then $(m \times b) * a=m \times b a-m b \times a=m \times b a$. Hence 
the mapping $m \otimes b \rightarrow m \times b$ induces an isomorphisms from $N_{0}$ onto $Q_{2} * 1$.

From this lemma and theorem 2.1, we have immediately

Theorem 3.3. Let $A / N$ be separable. Then 3-dimensional cohomology groups of $A$ are all zero if and only if $N_{0}$ is an $\left(M_{0}\right)$-module as an A-right module.

\section{REFERENCES}

[1] G. Hochschild, On the cohomology groups of an associative algebra, Ann. of Math., 46 (1945).

[2] - On the cohomology theory for associative algebras, Ann. of Math., 47 (1946).

[3] - Cohomology and representations of associative algebras, Duke Math. J., 14 (1947).

[4] M. Ikeda, On a theorem of Gaschütz, Osaka Math. J., 5 (1953).

[5] - On absolutely segregated algebras, Nagoya Math. J., vol. 6 (1953).

[6] Y. Kawada, On the cohomology theory of rings, J. Math. Soc. Japan, 1 (1948).

[7] H. Nagao and T. Nakayama, On the structure of $\left(M_{0}\right)$ - and $(M u)$-modules, forthcoming in Math. $Z$.

Added in proof : Recently T. Nakayama and M. Ikeda have proved jointly that if $n$-dimensional cohomology groups of $A$ are all zero then $A / N$ is separable. Using this theorem, Theorem 2.1 and 3.3 are improved as follows:

Theorem 2.1': Let $A$ be an algebra with unit element. Then n-dimensional cohomology groups of $A$ are all zero if and only if

i) $A / N$ is separable,

ii) $Q_{n-1}$ is an $\left(M_{0}\right)$-moalule as an A-right module.

Theorem 3. $3^{\prime}$ : Let $A$ be an algebra with unit element. Then 3-dimensional cohomology groups are all zero if and only if

i) $A / N$ is separable,

ii) $N_{0}$ is an $\left(M_{0}\right)$-module as an A-right module.

As is easily seen, Theorem $2.1^{\prime}$ is an actual generalization of Ikeda's theorem.

Mathematical Instilute,

Osaka University 